\title{
Mujeres y activistas: construyendo vejeces en narrativas patchwork ${ }^{1}$
}

\section{Nicole Mazzucchelli Olmedo}

(iD) https://orcid.org/0000-0003-1864-8553

Universidad Autónoma de Barcelona, España

Pontificia Universidad Católica de Valparaíso, Chile

nicole.mazzucchelli@pucv.cl

\section{María Isabel Reyes Espejo}

iD https://orcid.org/0000-0003-0454-0584

Pontificia Universidad Católica de Valparaíso, Chile maria.reyes@pucv.cl

\section{RESUMEN}

Los discursos sobre el envejecimiento de las mujeres mayores se han centrado en las problemáticas y dificultades que estas enfrentan, omitiendo el reconocimiento de sus recursos y potencialidades. Esta investigación tiene por objeto rescatar los significados de envejecer de mujeres activistas de la Agrupación Bordadoras por la Memoria, en Chile. Desde la gerontología feminista y la perspectiva narrativa, producimos narrativas patchwork que ofrecen nuevas versiones de ser mayor. Los resultados muestran la emergencia de narrativas sobre la vejez en pandemia, las distinciones de género, la sabiduría adquirida en esta etapa, y su experiencia de envejecer desde la acción politica, cuestionando las características con las que se describe tradicionalmente a las mujeres viejas. Se concluye que el ejercicio de construcción y cuestionamiento que las participantes realizan frente a su propia vejez desde su experiencia asociativa les

1 Este trabajo fue apoyado por la Agencia Nacional de Investigación y Desarrollo de Chile (ANID/Doctorado Nacional/2018-21180106), y es producto de la Tesis Doctoral de la primera autora. 
permite tensionar y disputar posiciones, transgrediendo la representación normativa de la vejez hegemónica.

Palabras clave: vejez femenina, gerontología feminista, acción política, mujeres mayores.

\section{Women and Activists: Constructing Old Age in Patchwork Narratives}

\section{ABSTRACT}

Discourses on the aging of older women have focused on the problems and difficulties they face, omitting the recognition of their resources and potential. This research reports the results of a research that rescues the meanings of aging of women activists of the Agrupacion Bordadoras por la Memoria -embroiderers' group for memory-, in Chile. From feminist gerontology and narrative perspective, we produce patchwork narratives that offer new versions of being older. The results show the emergence of narratives on pandemic old age, gender distinctions, the wisdom acquired at this stage, and their experience of aging from political action, questioning the characteristics with which old women are traditionally described. It is concluded that the exercise of construction and questioning that the participants carry out in front of their own old age from their associative experience, allows them to tense and dispute positions, transgressing the normative representation of hegemonic old age.

Keywords: female aging, feminist gerontology, political action, older women. 


\section{INTRODUCCIÓN}

En las últimas décadas, el estudio de la vejez y el envejecimiento de la población se ha convertido en objeto de interés en diversas disciplinas, más aún cuando se refiere a los países latinoamericanos por su acelerado proceso (Espinoza y Rodríguez, 2020). Uno de los aspectos más revisados es el de la feminización de la vejez, entendido como un fenómeno mundial, caracterizado por la amplitud de la presencia de mujeres en la población mayor, y el aumento de este porcentaje en la medida que se envejece (CEPAL, 2019). En este contexto, los países han implementado estrategias y políticas públicas específicas para dar respuesta a las demandas sociales de este grupo (Gonzálvez Torralbo, Larrazabal Bustamante y Lube Guizardi., 2020). Sin embargo, pese a estas acciones de reconocimiento, todavía un número amplio de personas mayores envejece en condiciones de pobreza y marginalidad social (Huenchuán, 2018). Esta situación se agrava para las mujeres que presentan niveles de exclusión social más elevados, debido a trayectorias de discriminación acumuladas a lo largo de sus vidas (Aguierre y Scavino, 2018). Así, la vejez es considerada como la etapa en la que se concentran y experimentan de forma más cruda las desigualdades de género y edad (ONU Mujeres, 2014).

A las dificultades ya descritas, se añaden dimensiones específicas del envejecer en el contexto de América Latina, que en palabras de Herminia Gonzálvez y Menara Lube (2020) habían resultado desapercibidas hasta hace unos años. Entre ellas destaca la continuidad del trabajo remunerado después de la jubilación en un escenario social caracterizado por la productividad neoliberal y la invisibilidad del trabajo de cuidados realizado por las mujeres. En el caso particular de Chile, el envejecimiento poblacional está marcado por la condición de género (INE, 2018), y en los últimos años se ha constatado un aumento en el número de los hogares unipersonales envejecidos y feminizados (Ortiz y Gonzálvez, 2017). Del mismo modo, los adultos mayores jubilados reciben una pensión inferior al salario mínimo legal, situación que es aún más precarizada para las mujeres (PNUD, 2017). Esta y otras situaciones quedaron en evidencia en los procesos de revuelta 
social en Chile durante el año 2019, cuando las personas mayores expusieron demandas específicas en torno a los sistemas de protección y seguridad social (Gonzálvez Torralbo et al., 2020).

De igual manera, en el contexto de crisis sanitaria por la COVID-19 que hemos enfrentado durante los últimos meses, las personas mayores han sido especialmente visibles, al ser catalogadas como principal grupo de riesgo. En ellas se focalizó una serie de regulaciones y prohibiciones, así como se han difundido representaciones edadistas que acentúan la discriminación de este grupo a propósito de su riesgo sanitario (Bravo-Segal y Villar, 2020). Asimismo, la literatura reciente ha reportado ampliamente las condiciones de vida en la vejez, y su relación con la pobreza y la exclusión social en los países latinoamericanos (Yuni, 2020). Estos discursos dominantes sobre la vejez y la población mayor actúan como guiones culturales que orientan las representaciones con las que vinculamos a las personas mayores (Serrat y Villar, 2015) y que, en particular, para el caso de las mujeres, las asocian a la fragilidad, pasividad y debilidad, caracterizándolas como un grupo homogéneo y con abundantes problemáticas (Gonzálvez y Lube, 2020).

Coincidentemente, en los últimos años ya se venía incrementando el interés por conocer el grado de bienestar y la propia experiencia subjetiva en la vejez (Gonzálvez, 2018). Es así como la indagación en los modos específicos en los que las mujeres envejecen comenzó a ser objeto de interés en el ámbito público —institucional y científico (Navarro y Danel, 2019)_, aunque los estudios desde las ciencias sociales aún resultan insuficientes (Ramos, 2016). Por lo general, el conocimiento gerontológico había sido reducido a características homogéneas y androcéntricas de la población mayor, sin explorar la especificidad de la vejez femenina (Freixas, 2008). Así, el abordaje en torno al género y vejez desde enfoques críticos, comenzó a ser un campo en incipiente desarrollo (Ramos, 2018). En él, destacamos algunas investigaciones, como la organización social de los cuidados y las políticas públicas (Gonzálvez Torralbo, 2018; Gonzálvez Torralbo et al., 2020), las violencias de las mujeres mayores desde una perspectiva interseccional (Mazzucchelli y Adaos, 2019) y la problematización del envejecimiento desde un marco gerontológico feminista (Navarro y Danel, 2019). No obstante, pese a estas iniciativas se puede observar que la indagación se ha concentrado mayormente en las mujeres mayores y sus vulnerabilidades, y en menor medida se ha puesto énfasis en sus potencialidades y agencia (Gonzálvez, 2018; Ramos, 2017). 
Una de las dimensiones que ha permanecido ausente en el debate es el activismo y asociatividad de las mujeres. En general, los estudios en la población mayor no se han interesado por las particularidades que adquiere la participación desde el género (Serrat y Villar, 2020), aunque algunas investigaciones han reportado cierta disparidad entre las agencias de hombres y mujeres, como producto de sus trayectorias de vida generizadas (Ramos, 2017). En este sentido, el activismo de las mujeres mayores ha sido mayoritariamente estudiado en torno a las contribuciones que han realizado en etapas previas a la vejez, omitiendo con ello las experiencias y prácticas que despliegan en esta etapa de vida. Del mismo modo, las investigaciones se han focalizado en trayectorias individuales y recursos personales, restando valor al carácter relacional y psicosocial de la experiencia de envejecer (Chazan, 2016; Wray, 2004). En este contexto, es posible rastrear el desarrollo en ciernes de investigaciones que exploran la vejez de mujeres mayores de la mano de la acción colectiva, principalmente desarrolladas en el contexto anglosajón. Estos estudios exponen la importancia del proceso de identificación y reconocimiento que confluye grupalmente, y cómo la experiencia asociativa es altamente valorada en los proyectos de vida de las mujeres al envejecer (Chazan, 2016; Sawchuk, 2009; Wray, 2004-2007). Adicionalmente, muestran de manera ejemplar cómo las mujeres mayores contribuyen a los procesos de transformación social y al desarrollo de sus grupos y comunidades de referencia, renovando sus repertorios de acción en la medida que envejecen (Charpentier, Quéniart y Jacques, 2008; Charpentier y Quéniart, 2017).

En este artículo ofrecemos una contribución en esta línea, indagando acerca de la forma en que las mujeres mayores activistas vivencian su vejez desde su experiencia asociativa en tiempos de pandemia global. De este modo, describimos los significados de envejecer de un grupo de mujeres mayores de la colectiva Bordadoras por la Memoria de Valparaíso, en el contexto de crisis sanitaria en Chile. En este escenario, observamos cómo los vínculos y las relaciones de sororidad que se establecen entre mujeres mayores contribuyen a resignificar sus propias experiencias de envejecimiento (Pérez, Rábago, Castillo, Schoham, Zamora, López, 2018). La apuesta, en este sentido, contribuye a tensionar el conocimiento hegemónico y esencialista del ser mujer mayor, que suele representarlas como frágiles-pasivas y dependientes (Freixas, 2008), favoreciendo el hecho de rescatar y visibilizar los sentidos que las mujeres van otorgando a su vejez por medio de la técnica del patchwork. De esta forma, producimos narrativas desde múltiples voces, que no se reducen a un pensamiento único/homogéneo, (re)presentando una diversidad de puntos de vista entrelazados en un mismo discurso (Biglia y 
Bonet-Martí, 2009). Así, las temáticas abordadas surgen a partir de los aprendizajes que nos proporcionaron los encuentros con las participantes y permiten poner en valor sus saberes expertos y su pericia.

Para dar respuesta al propósito de este trabajo, en un primer momento describiremos el marco que lo sostiene teórica y políticamente, de la mano de la gerontología feminista. Posteriormente, abordaremos el método-proceso con el cual se llevó a cabo el estudio, destacando la perspectiva narrativa y sus potencialidades en el campo gerontológico. En un tercer momento, daremos cuenta de los principales resultados de la investigación a través de narrativas desde múltiples voces. Finalmente, compartiremos algunas reflexiones desde las que se sitúan algunos desafíos para el campo de investigación de las vejeces generizadas en el marco de sus agencias políticas asociativas.

\section{MUJERES MAYORES: UNA APROXIMACIÓN EN CLAVE FEMINISTA}

En las últimas cinco décadas, la vejez y el género se han posicionado como campos de estudio científico, aunque ambos han avanzado por caminos separados (Yuni, 2019). En materia de derechos humanos, la agenda internacional ha promovido la visibilización de los problemas y necesidades que aquejan a las mujeres mayores, pero estos no han logrado proporcionar una base adecuada que incorpore sus trayectorias de vida (Ramos, 2010, 2018). Así, la segregación por motivos de edad, sumada a los estereotipos del sexo, agudiza aún más los problemas sociales de las mujeres mayores al situarlas como beneficiarias de los sistemas de producción, omitiendo, con ello, su papel de sujetos contribuyentes (Nieve, 2010). De esta manera, si bien la investigación gerontológica se ha consolidado, también ha naturalizado y ampliado las diferencias y las desigualdades que se articulan entre el género y la edad (Yuni, 2019).

Desde la gerontología crítica ${ }^{2}$, entendida como un enfoque multiforme que se distancia de los modelos convencionales-biomédicos para situar a las personas mayores — más allá de la asistencia social—, como agentes con plenos derechos (Paola, 2015), ha habido consecuencias en el estudio de las mujeres mayores y

2 Se agrupan en la denominada gerontología crítica, distintas perspectivas en el campo de los estudios de la edad, como la gerontología feminista, las que rechazan las categorías binarias y homogéneas que ubican a las personas mayores desde análisis dicotómicos —autónomodependiente, productivo-improductivo-, abordando problemáticas que en la gerontología tradicional no han tenido cabida, como la redistribución de recursos y la justicia social (Katz, 2014). 
sus experiencias de envejecer, desarrollando cuestionamientos en torno al tratamiento con el que se ha abordado a este grupo. Por una parte, durante décadas en la gerontología, el género fue considerado tan solo una categoría médicobiológica, naturalizando ciertas prácticas como femeninas sin advertir que su construcción ha sido producida en un sistema patriarcal (Yuni, 2019). A su vez, se han construido imágenes y representaciones de la vejez que responden más bien a un modelo hegemónico de vejez exitosa, el que da cabida a vejeces deseables $\mathrm{y}$ visibles y, en menor medida, a aquellas subordinadadas o silenciadas (Yuni, 2020). Un ejemplo de esto lo encontramos en el indicador global de envejecimiento «Active Ageing Index» (AAI) elaborado por la UNECE (United Nations Economic Commission for Europe) que intenta describir las condiciones en las que envejecen las poblaciones (Zaidi et al., 2013), sin atender al carácter situado ni las particularidades de envejecer desde otros territorios. Asimismo, los modelos convencionales se han centrado en el estudio de las dimensiones individuales asociadas a la vejez, invisibilizando las condiciones opresivas y desiguales en las que se envejece (Mazzucchelli, 2019), restando así atención al carácter psicosocial de la experiencia.

En respuesta a estas limitaciones, las perspectivas no convencionales en gerontología - como la gerontología feminista - buscan visibilizar la construcción social de los significados, analizando los efectos que la diferencia sexual y los patrones de dominación han tenido y tienen en las experiencias de vida de las mujeres (Calasanti, 2010; Freixas, 2008; Freixas, Luque y Reina, 2012). Igualmente, en los últimos años, desde los feminismos latinoamericanos se han instalado discusiones que traen al debate miradas altamente contextuales que problematizan la estrecha relación entre los cuidados y las transformaciones en los cursos de vida de las mujeres desde una manera transgeneracional (Gonzálvez y Lube, 2020). Con ello, se advierte que debemos apostar por pensar a las mujeres mayores desde sus múltiples posiciones, donde la intersección género y edad son la base para otras articulaciones posibles en torno a la opresión (Navarro y Danel, 2019). Así, la gerontología feminista nos invita a reconocer también que las mujeres mayores han enfrentado trayectorias de discriminación diversas, donde las experiencias asociativas han favorecido el despliegue de su agencia y enfrentamiento de estas (Wray, 2003).

En este contexto, la participación social en la vejez ha sido una dimensión ampliamente promovida por su incidencia positiva en el bienestar de personas mayores y sus comunidades (Gonzalez, Matz-Costa y Morrow-Howell, 2015). No obstante, los estudios que abordan la participación política de las mujeres 
mayores desde una perspectiva de género aún son reducidos (Serrat y Villar, 2020) y tienden a describir la participación como una acción acrítica y despolitizada (Zubero, 2018). Como efecto, se ha tendido a desatender e invisibilizar las trayectorias de acción política de las mujeres mayores a lo largo de sus vidas y que, en particular en los países del Cono Sur, se han desarrollado comprometidamente desde sus contextos cotidianos-comunitarios (Valdés, 1986). Recordemos que ellas pertenecen a la generación que protagonizó la defensa a los derechos humanos, la resistencia a las dictaduras y la reconstrucción de la democracia (Kirkwood, 2010; Sapriza, 2009). Sin embargo, los estudios en torno a las mujeres mayores se han centrado en sus actuaciones en el pasado, ignorando la experiencia que hoy, al ser mayores, desarrollan en términos políticos.

Esta investigación pretende avanzar en este vacío, profundizando en las vivencias y significados que las mujeres atribuyen a la vejez, desde sus propias voces y experiencia colectiva. Así, proponemos tensionar las representaciones convencionales de vejez, focalizadas en experiencias individuales, para rescatar el carácter asociativo y compartido de la experiencia de envejecer de la mano de mujeres activistas. De esta manera, apostamos por visibilizar agencias y resistencias con que las que las mujeres subvierten sus propias experiencias y significan sus procesos de envejecimiento, en un escenario especialmente relevante y restrictivo para las personas mayores dada la crisis sanitaria global, en tiempos de pandemia y post pandemia.

\section{EL MÉTODO: NARRATIVAS Y SENTIDOS}

De la mano de los saberes feministas y la perspectiva narrativa, proponemos una diada teórica-metodológica en el abordaje de los significados que mujeres mayores activistas otorgan a su vejez. Las narrativas están presentes en la vida cotidiana y, a través de ellas, articulamos la dimensión cultural y personal, construyendo significados para poder comprender y mediar la realidad (Gergen y Gergen, 1983). Compartimos que lo narrativo no puede ser reducido a un conjunto de métodos o técnicas, pues ello despolitiza esta perspectiva y reduce su potencial de cambio (Bamberg, 2012). De esta forma, en nuestro interés por aprender de las mismas protagonistas y poner en valor sus conocimientos expertos, las narrativas ofrecen un lugar privilegiado para explorar las intersecciones de la vida personal y social. Así, adscribimos a la construcción de narrativas como un método-proceso de investigación que incluye un posicionamiento ético-político específico que 
reconoce que, en el encuentro entre diferentes subjetividades, se va conformando la narrativa en sí misma (Biglia y Bonet-Martí, 2009).

Desde este lugar, apreciamos el potencial transformador propio de la narrativa, no solo en su contenido, sino en las relaciones y la capacidad de acción que los propios sujetos tienen en ellas (Schöngut y Pujol, 2015). Aproximarnos a las narrativas nos permite interpretar historias y, al mismo tiempo, crear nuevos significados en conjunto, involuntarios e impredecibles, y que no pueden ser atribuidos a las intenciones de ninguna persona en particular (Cabruja, Iñiguez y Vásquez, 2000). Así, a través del proceso de construcción colectiva entre diversas subjetividades se favorece la producción de nuevas narrativas, o de narrativas alternativas que se configuran como una acción que enfrenta a las relaciones de poder - reforzadas desde los discursos dominantes-, contribuyendo a recrear nuevas realidades a través de narraciones subversivas y situadas como una acción conjunta (Biglia y Bonet-Martí, 2009). Este trabajo da cabida también a aquellos elementos - ignorados por considerarse «acientíficos»-, como las emociones, las subjetividades y las corporizaciones (Biglia y Bonet-Martí, 2009) y que, por lo general, suelen pasar inadvertidos para la gerontología tradicional. Así, la perspectiva narrativa contribuye ampliamente al campo de los estudios de envejecimiento, permitiéndonos profundizar en los propios relatos con los que los actores dan cuenta de su experiencia de vejez. Asimismo, permiten distinguir los discursos dominantes/ hegemónicos que construyen sus representaciones (Villar y Serrat, 2015) y caracterizan a las mujeres mayores, para así disputarlos desde narrativas situadas.

La investigación, aprobada por el Comité de Ética y Bioseguridad de la Universidad de afiliación de las autoras, fue desarrollada durante mayo del año 2020 y duró hasta enero del año 2021, en la ciudad-puerto de Valparaíso, capital cultural y legislativa de Chile. Trabajamos con las mujeres mayores de la agrupación Colectiva Bordadoras por la $\mathrm{Memoria}^{3}$, grupo compuesto solo por mujeres que, desde el año 2016, se reúne de manera periódica en el Centro Cultural de la ex cárcel en Valparaíso. Las Bordadoras comparten el interés por la justicia social, los derechos humanos y la mantención de la memoria, para que los hechos ocurridos en la dictadura cívico-militar de Pinochet no se olviden y puedan servir

La Colectiva se inspira en la artesanía comunitaria de arpillera, que surgió en el contexto de la dictadura militar en Chile. A través de sus arpilleras, las mujeres comunicaban y denunciaban la búsqueda de sus familiares detenidos desaparecidos y la violación a los derechos humanos acaecidos durante la dictadura. En honor a estas mujeres y sus reivindicaciones, la agrupación hizo un esfuerzo de distinción y eligió la técnica del bordado como práctica política de su activismo por la memoria. 
como aprendizaje para las generaciones venideras. Entre sus principales acciones políticas se encuentra el bordado. A través de sus obras/bordados recuerdan colectivamente, expresan posicionamientos y denuncias y revindican derechos. En una de sus últimas exposiciones bordaron la nueva Constitución de Chile y las dimensiones que, como colectiva, consideran que debiese integrar. También participan de otros espacios de acción política, adhiriendo a las reivindicaciones de movimientos sociales que promueven los derechos sociales de las mujeres, la erradicación de la violencia, así como la defensa por los derechos ambientales y de los territorios.

A continuación, compartimos una breve descripción de cada una de las participantes de la agrupación. Para preservar las identidades de las mujeres entrevistadas en los resultados expuestos. Sus nombres fueron omitidos y reemplazados por un seudónimo.

Tabla 1. Caracterización de las participantes

\begin{tabular}{|c|c|c|c|c|}
\hline Participantes & Edad & Oficio-profesión & $\begin{array}{l}\text { Estado } \\
\text { civil }\end{array}$ & $\begin{array}{c}\text { Participación en otras } \\
\text { organizaciones }\end{array}$ \\
\hline Gabriela & 71 & Modista - jubilada & Casada & $\begin{array}{l}\text { Agrupación de pensio- } \\
\text { nados, Colectivo 8m }\end{array}$ \\
\hline Michelle & 73 & Profesora - jubilada & Casada & $\begin{array}{c}\text { Militante Partido } \\
\text { Comunista y otras agru- } \\
\text { paciones }\end{array}$ \\
\hline Laura & 63 & Profesora - jubilada & Separada & $\begin{array}{l}\text { Agrupación cine y } \\
\text { narrativas y otras }\end{array}$ \\
\hline María Cecilia & 68 & Trabajadora social & Casada & $\begin{array}{c}\text { Militante Partido } \\
\text { Comunista y otras agru- } \\
\text { paciones }\end{array}$ \\
\hline Aylen & 69 & Enfermera - jubilada & Casada & $\begin{array}{l}\text { Agrupación Cine y } \\
\text { Narrativas y otras }\end{array}$ \\
\hline Reinalda & 68 & $\begin{array}{c}\text { Dueña de casa - arte- } \\
\text { sana }\end{array}$ & Viuda & $\begin{array}{c}\text { Agrupación de mujeres, } \\
\text { Familiares de detenidos } \\
\text { desaparecidos, y otras. }\end{array}$ \\
\hline
\end{tabular}

Consideramos importante destacar que el trabajo de campo se llevó a cabo en un contexto sociosanitario complejo a propósito de la crisis por la COVID-19, que impactó en los modos de conducir y adaptar el diseño de investigación. En 
este sentido, si bien no podemos eludir la relación de poderes (investigadorasparticipantes) presente en un proceso de investigación, nos propusimos mantener la apertura y flexibilidad metodológica a las necesidades de las participantes y sus propias vivencias, al ser uno de los grupos sociales más afectados por el contexto de salud y las restricciones impuestas para el manejo de la pandemia. De esta manera, readaptamos las entrevistas a formato remoto, realizando entre una y dos entrevistas por cada participante, las que tuvieron una duración promedio de 2,5 horas. Así, fuimos reconduciendo los temas abordados a propósito de las inquietudes y experiencias que las propias mujeres fueron exponiendo. Este proceso favoreció la creación de espacios de empatía y confianza con las participantes, promoviendo un diálogo que permitió compartir vivencias personales e íntimas. Los resultados que aquí se presentan constituyen un esfuerzo por visibilizar sus experiencias.

\section{LA TÉCNICA DEL PATCHWORK}

En este trabajo, consideramos la construcción de narrativas como un métodoproceso de investigación, incorporando la producción de narrativas patchwork ${ }^{4}$, que en palabras de Bárbara Biglia (2005), no es otra cosa que la readaptación a los textos escritos de los que nuestras abuelas hacían con las telas (p. 162). En afinidad con los conocimientos situados (Haraway, 1988), y con el propósito de que la metodología surgiera a través del trabajo empírico, no se diseñó una propuesta analítica con antelación en el diseño, sino que esta fue sensible al encuentro con las participantes (Biglia y Bonet-Martí, 2009). Para construir las narrativas patchwork, en el trabajo empírico transitamos por diferentes etapas, que se detallan a continuación:

1) En primer lugar, realizamos entrevistas biográficas (individuales) a las mujeres mayores de la agrupación (entre los 63 y 74 años de edad). Estas se realizaron en formato remoto a través de la aplicación Zoom.org, y tuvieron una duración promedio de dos horas y media. Luego, estas fueron transcritas íntegramente.

2) Posteriormente, realizamos una lectura exhaustiva de cada uno de los textos producidos en los encuentros, sensibilizándonos con la historia narrada, los personajes, la trama, los hitos, motivaciones, etc. (Bertaux, 2005; Piña, 1999).

4 La técnica del patchwork - o almazuela, en castellano - consiste en crear una pieza de telatejido uniendo diferentes telas, lanas o materiales, Jugando con la creatividad y la imaginación, van emergiendo nuevas creaciones y propuestas de ensamblajes. 
3) A partir de la propuesta analítica singular-transversal para los relatos (Cornejo, Faúndez y Besoain, 2017), se continuó explorando los textos producidos, articulando las dimensiones personal/subjetiva y político/social (Cornejo, Mendoza y Rojas, 2008). Para ello, realizamos primero una codificación de cada entrevista, categorizando cada uno de los textos (con foco en lo singular), y posteriormente identificamos las semejanzas, tensiones y desplazamientos de cada corpus (para abordar su transversalidad).

4) A partir de estas categorías, que describían cada dimensión en el análisis transversal, construimos el patchwork que encadena y entrelaza diferentes fragmentos otorgados por las participantes, a cada eje analizado de las entrevistas. Estas narrativas transitan por diferentes significados otorgados a la vejez, contribuyendo a la elaboración de un discurso donde se despliegan diferentes voces.

Las narrativas patchwork que se presentan aplican esta técnica, mencionando con seudónimos a las autoras de cada frase para mantener la autonomía de las diferentes voces y mostrar puntos de vista difractarios (Haraway, 1988), es decir, produciendo comprensiones y explicaciones sobre los fenómenos que tensan, diversifican y complejizan los discursos dominantes de la vejez. Asimismo, incorporan entre paréntesis el uso de conectores para otorgar un sentido más completo al texto producido (Biglia y Bonet-Martí, 2009).

Figura 1. Exposición «Los derechos que queremos en la nueva Constitución», $2020^{5}$.

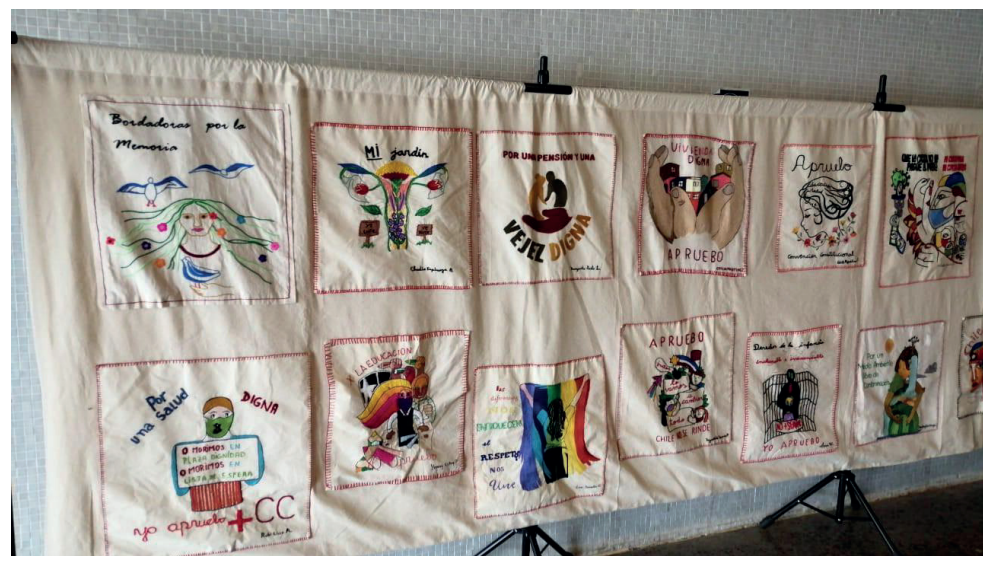

Tapiz que representa el voto por el «Apruebo» una nueva Constitución en Chile. A través de sus bordados, dan cuenta de las diferentes temáticas que debe incluir la constitución, para avanzar hacia un país más justo e inclusivo con todos y todas. 


\section{VEJECES EN PATCHWORK: PRÁCTICAS DE ESCRITURACOMPARTIDA}

A continuación, se presentan las narrativas colectivas construidas con las mujeres mayores. Se han organizado en cuatro narrativas ficcionales que dan cuenta de sentidos y significados atribuidos a su vejez y a su experiencia de envejecer. En cada narrativa se han extraído frases importantes relativas a la dimensión abordada, dando vida a los tejidos patchwork que se comparten.

\section{i. Envejecer en pandemia}

[La pandemia ha sido] horrible, primero porque tenían que estar encerrados los viejos, y segundo, porque de verdad uno empieza a preguntarse o a cuestionarse, iya poh!, ya viví suficiente [...] Uno siente que ya no tiene futuro, que el futuro es hoy día, o a lo mejor mañana sábado, o pasado mañana domingo. Ese es tu futuro (Michelle). Yo no quería estar quedándome en casa $[\ldots]$ yo siempre he tenido la idea de que cuando uno es viejo y se queda en la casa, se atrofia y se pierde como persona (Aylen). [Entonces] yo digo: «Pucha, me llegó la vejeztud de una», porque una perdió el ritmo de hacer muchas cosas. Yo tenía todos los días, todos los días ocupados [...] mañana o tarde, pero siempre había alguna actividad (Gabriela), pero eso de no poder hacer [...] porque está todo suspendido [...] Entonces he hecho varias cosas acá en la casa, pero me siento en la mesa a trabajar, después en el sillón, después a la cama (risas) [...] Entonces, la falta de ejercicio ha causado, así como estragos en mis articulaciones: me cuesta pararme, salgo a caminar y me canso rápido (Aylen). [Lo más difícil de la pandemia] ha sido estar sola $[\ldots]$ yo soy muy social. Me encanta tener la casa llena de gente, que vengan los jóvenes, los viejos y la gente que quiera venir. Creo que me ha pegado fuerte por esa razón más que nada [aunque pienso que] todas las cosas tienen un porqué [...] Yo creo que el mundo no daba para más así como estaba, yo creo que esto va a servir para reflexionar (Reinalda) [...] Hay gente que nunca se detuvo a pensar y a lo mejor está tomando un poquito más de caldo de cabeza y está pensando en qué tan mal lo hicimos como sociedad para llegar a ser tan egoístas como lo estamos siendo ahora (Laura).

Las participantes dan cuenta de una asociación directa entre el comenzar a sentirse «mujeres mayores» o mujeres viejas con el confinamiento y la declaración de estado de emergencia sanitaria. Tempranamente, la Organización Mundial de la Salud (OMS, 2020) declaraba que el principal grupo de riesgo estaba constituido por personas mayores. Así, el contexto pandémico, se configura como un hito central que modificó su percepción del tiempo, alteró sus rutinas e impactó en el desarrollo de sus actividades y relaciones. Con la indicación de distanciamiento 
social y la permanencia en casa, emerge la posibilidad de un futuro incierto y se desarrolla un cuestionamiento sobre sus proyectos de vida. Los discursos que se presentan a la población —en los medios de comunicación, por ejemplo- como acontecimientos de gran envergadura e impacto influyen en su comprensión y representación sobre un fenómeno (Nespereira-García, 2014) y, en particular, el abordaje que ha tenido el COVID-19 ha promovido la circulación de discursos edadistas que destacan la discriminación por motivos de edad (Bravo-Segal y Villar, 2020). Esta narrativa muestra que las mujeres mayores activistas asocian la vejez con la permanencia en casa, con una mayor inactividad, y la disminución de sus acciones de implicancia social. Así, el modelo convencional de envejecimiento activo / exitoso (OMS, 2000) que describe las dimensiones que favorecen una vejez saludable - como la participación social y la actividad física- son representadas como elementos importantes para las participantes, y que han dejado de estar presentes en su cotidianidad en contextos de pandemia, situación que relatan como una experiencia negativa.

Por otra parte, el estado de emergencia impacta en sus rutinas debido a una serie de prohibiciones y restricciones que aplican de forma exclusiva para este grupo etario (MINSAL, 2020). Estas enumeran las actividades que pueden (o no) desarrollar y que se concentran exclusivamente en el espacio doméstico, lo que influye en el reconocimiento de molestias o lesiones físicas que las conectan con su corporalidad, al disminuir las actividades físicas y de esparcimiento que desarrollaban fuera de casa. Las participantes señalan que, antes de la restricción, su vida cotidiana se organizaba en diferentes espacios y que sus actividades daban sentido a sus experiencias. Estos cambios impulsan reflexión y el desarrollo de procesos subjetivos (Piña, 1998-1999) que las hacen resituar su condición de mujeres mayores, redefiniéndose como seres en el mundo. La imposibilidad de relacionarse con otras personas y de poder compartir físicamente es otra de las causas que han afectado su salud mental y emocional. Esta experiencia también las ha impulsado a resignificar sus vínculos, impactando en su subjetividad: se conectan con sus añoranzas, motivaciones y expectativas, ubicando el devenir como un tránsito incierto, sobre el cual se reflexiona y problematiza (Arfuch, 2007).

En el estado de confinamiento, también han desarrollado cuestionamientos en torno al futuro de la humanidad, la sociedad, y la responsabilidad que, como seres humanos, tenemos en la situación actual. Comparten la idea de un futuro gris y la evaluación de sus trayectorias de vida desde un presente que va más allá de la contingencia. Así, sus procesos reflexivos enfrentan los discursos hegemónicos sobre el COVID-19 y problematizan más ampliamente el devenir, con un foco 
que trasciende la mirada sobre el individuo, el contagio y la pandemia. En su narrativa, estas mujeres dan cuenta de lo que Donna Haraway (2019) ha referido como el cuidado en tiempos de urgencia/crisis y a la respons-habilidad, entendida como un estar dispuestos a responder y enfrentar la realidad de un planeta que está muriendo, por lo que es necesario generar nuevas relaciones con lo que llamamos naturaleza. Esta narrativa patchwork da cuenta de una preocupación por la humanidad y por el futuro legado a otros y otras que es afín a la trayectoria de lucha por la que estas mujeres se han movilizado a lo largo de sus vidas.

\section{ii. Distinciones sexo-género}

El hombre se jubila y como que ya no tiene la razón pa'vivir; porque el hombre, pa'la cabeza del hombre, el trabajo es el fin de su existencia. En cambio, la mujer siempre tiene una razón pa'vivir. Está su casa, están los nietos, están los hijos (Michelle). [Las mujeres tienen] una carga social distinta. Llevas contigo responsabilidades que no te puedes deshacer $[\ldots]$ de la responsabilidad de madre; eres mamá hasta que te mueres. Y el apoyo incondicional al compañero; a todo poh', a lo que te rodea, a todo [...] el desgaste físico y mental es mucho mayor... pero aunque estés más desgastada, eres mucho más autosuficiente que el varón [...] (Reinalda).

[Los hombres] se ponen más dependientes, quieren ser servidos, se ponen más machistas, más idiotas. Ellos ya no quieren salir [...] Dejan de estar activos. Nosotras somos como al revés, lo veo así (Laura). [Hay hombres] viejos que no se saben freír un huevo, poh (Gabriela). [Cuando] se les murió la señora no saben llevar la vida solos, lo único que buscan es una compañera nueva $[\ldots]$ antes de andar extrañando [...]. [En cambio] se le muere el marido a una señora, pasan su etapa de duelo y después es una mujer que ha ganado [...], no sé, y se ven regias, se arreglan, se cambian el estilo, se pintan el pelo. La mayoría de las mujeres no, poh', lo único que quieren es su independencia, estar solas y disfrutar, ¿te fijas? Eso es en el común que yo observo, y los estudios también lo dicen (María Cecilia).

Las participantes afirman que el envejecimiento se expresaría de forma diferente en hombres y mujeres, como producto de cualidades específicas del ser mujer vinculadas con el cuidado, las relaciones y el soporte de la vida. Refieren que envejecen en mejores condiciones que los hombres, contradiciendo así la literatura que ha caracterizado que las mujeres mayores viven su vejez en condiciones más precarias como producto de trayectorias de discriminación diversas (Aguierre y Scavino, 2018). Una de las dimensiones que destacan al compararse con los hombres es que estos, al jubilarse, pierden el sentido y propósito de su 
vida, lo que los lleva a tener dificultades para valorar el tiempo en casa o el mayor tiempo libre. Por el contrario, las mujeres, al jubilarse, valoran las dimensiones en torno al cuidado, la crianza y las relaciones interpersonales, significándolas como actividades que aprecian en su vejez, junto con disponer de mayor tiempo personal para sus propios proyectos.

Esta dimensión se condice con algunos estudios que han descrito el empoderamiento de las mujeres en la vejez como la conquista de espacios, tiempos y el rescate de proyectos personales (Pinazo et al., 2019). Las mujeres significan su vejez como una etapa en la que pueden permitirse pensar más en ellas y dedicar más tiempo para su autocuidado y bienestar. El manejo del tiempo y de sus rutinas son una conquista personal que se aprecia y significa positivamente. No obstante, también implica el ejercicio de una negociación constante con ellas mismas, pues permitirse nuevos modos de vivir no está exento de las responsabilidades y mandatos sociales tradicionales de género que continúan tensionando sus decisiones. De esta forma, sus experiencias van ejerciendo transgresiones de lo socialmente esperado en el campo de la edad, lo que, además, les permite cuestionar los preceptos de buenas costumbres con los que se vincula a las mujeres mayores (Freixas, 2008).

Por otra parte, el retiro laboral de los hombres impacta en sus relaciones al interior del hogar, lo que puede incrementar prácticas machistas, ya que estos se desligarían completamente del trabajo doméstico. Esta situación no cambiaría diametralmente en las mujeres, pues si bien el trabajo doméstico ha sido parte de sus trayectorias vitales - y continuarían desarrollándolo-, gestionan y organizan sus tiempos desarrollando nuevos intereses y utilizando más tiempo en otros proyectos. A su vez, una de las temáticas frecuentemente estudiada en la vejez tiene que ver con la soledad y viudez de las personas mayores como dimensiones que afectan negativamente su envejecimiento (Montes de Oca, 2011). Coincidentemente, las mujeres mayores refieren que los hombres tendrían menos capacidad de estar solos, sin pareja, al enviudar, por no saber vivir de forma independiente, mientras que ellas mantendrían su independencia y autonomía emocional, pudiendo sobreponerse y resignificar su vida, desarrollando mecanismos para enfrentar de mejor forma las pérdidas. Las participantes pertenecen a una generación condicionada por un patrón sociocultural de dominación de género que limitó su autonomía y libertad, ubicándolas en la esfera privada y de reproducción (Freixas, 2018). Pese a ello, las bordadoras dan cuenta de un cuestionamiento a la idealización del matrimonio, la vida en pareja y los exclusivos roles de reproducción y cuidados atribuidos a las mujeres. 
Las participantes describen una vejez que diferencia las experiencias de hombres y mujeres, manifestando que sus vidas han sido más desgastantes y difíciles debido a sus responsabilidades vinculadas con el trabajo doméstico y de cuidados, labor que sido invisible y con poco reconocimiento social, dada la naturalización de su ejercicio «por ser mujeres» y al concepto mismo de feminidad (Aguirre y Scavino, 2018). Distinguen un ordenamiento sexo/género en las prácticas y representaciones culturales por las cuales transitaron en sus vidas; sin embargo, pese al reconocimiento de trayectorias diferenciadas producto del género, sostienen que las mujeres enfrentarían la vejez en mejores condiciones que los hombres, atribución que - como ya hemos señalado - no se condice con la representación que se sostiene desde el campo científico. Esta caracterización de una vejez diferenciada se sostiene en argumentos vinculados con la construcción de lo propiamente «femenino», como la maternidad, los cuidados y los afectos, instancias culturales desde las cuales se justifica la existencia de una identidad genérica estable sobre la base de su envejecimiento. Así, la diferencia entre hombres y mujeres podría ser entendida no por un conjunto de esencias que prexistirían en cada persona, sino por un sistema complejo de diferencias culturales, sociales, históricas y psicosociales donde se constituye nuestra experiencia (Barret, 1990).

Finalmente, en su vejez las mujeres transforman sus rutinas tanto materiales (cambio en el desarrollo de roles o actividades) como en una dimensión simbólica. La construcción subjetiva y la relación que establecen con su propia experiencia les permiten brindar más atención al cuidado de sí mismas, así como a la aceptación de sus trayectorias y experiencias. La subjetividad de las personas es un proceso dinámico y en constante transformación, que se construye socialmente desde la experiencia situada (Esteban, 2015). Así, la vivencia de la vejez es contextual y está habitada por las mujeres y recreada desde sus prácticas, donde sí se reconocen el género y la edad como factores claves en su elaboración identitaria. Esta no permanece estable y fija, sino que es interpelada a través de las expectativas y cambios que las mujeres van desarrollando a lo largo de sus vidas (Ramos, 2018).

\section{iii. Saberes femeninos}

La riqueza del ser humano es esa: de llegar a una edad y tener la capacidad de análisis, de reflexión, porque aprendiste de la vida, no porque fuiste a la universidad ni porque hayas estado con gente [...]. Lo que recoges, lo que te 
queda, lo que te hace sabio [...] es lo que has recogido a través de tu vida, de tus experiencias; eso es lo que le queda a una persona sobre sesenta (María Cecilia). [Estas experiencias te preparan] para lo que viene, que es la parte más difícil de la vida, que hay que aprender a estar con uno misma, que hay que vivir en armonía, en tranquilidad (Aylen). [Entonces] estoy en esa etapa de tratar de conciliarme con ese tiempo; ¿cómo te dijera?, acoger en el fondo a esa Reinalda chica que se vio desamparada, que se vio vulnerable, qué sé yo, y acogerla, acogerla ya como una mujer vieja, como una mujer grande; y hacerla sentir protegida [...] (Reinalda). [Una] tiene una cajita, así como donde va guardando toda la sabiduría que puedes ir recogiendo con las experiencias de vida desde el momento que naces (María Cecilia). La persona adulta tiene la experiencia para demostrar lo que pasó, y lo que puede pasar (Gabriela). [Y una está] tratando de dejar algo, aunque no sean cosas físicas, pero dejar algo; alguna enseñanza (Laura).

La etapa de la vejez para las mujeres mayores activistas es valorada como un momento en el cual experimentan cambios en su autopercepción y en la constitución del yo. Esta es descrita como un proceso de mayor introspección y evaluación de sus experiencias de vida, las que les permiten incorporar aprendizajes y valorarlos en esta etapa. A su vez, desarrollan una distinción entre la educación formal - la que proveería de un tipo de conocimiento o institución- $\mathrm{y}$ el aprendizaje de la «vida misma», que es el que les permite una comprensión más compleja de lo vivido. Este argumento es sus narrativas es iluminador si consideramos que las mujeres han sido un grupo social históricamente silenciado, escasamente reconocido (Del Valle, 1999) e ignorado por los discursos oficiales que han reproducido saberes masculinos por sobre los saberes femeninos (Freixas, 2015) como los únicos válidos y valiosos. Por lo contrario, en este patchwork se reivindican los saberes femeninos particulares, que tendrían la capacidad de orientar y apoyar a otros y otras, ubicando así a las mujeres mayores como referentes de un conocimiento transmisible/heredable.

Las mujeres significan su vejez como un constante devenir que las va aproximando a una etapa de mayor fragilidad-dependencia cercana a la muerte. Este momento se representa como una instancia difícil y dura de afrontar que les exige estar preparadas emocionalmente para llegar al final de la vida. En la vejez existen amenazas al sentido de continuidad personal que pueden producirse por las pérdidas y adaptaciones que deben afrontar las personas mayores, por tanto resulta importante la reflexión autobiográfica que estas pueden desarrollar (Villar y Serrat, 2015). Las mujeres añoran llegar al término de su vida en «armonía y tranquilidad» y describen algunas dimensiones analíticas que se debe elaborar en 
la vejez para afrontar esta experiencia con mayor tranquilidad. Entre ellas está el reconciliarse con su propia historia, con su pasado, y el reconocerse y aceptarse. Este trabajo puede estar vinculado con lo que algunos autores han descrito como tareas propias de la vejez o «tareas de integridad», que implicarían hacer una revisión vital de los acontecimientos vividos, reflexionando sobre el sentido de la vida y haciendo un balance de esta (Erikson, Erikson y Kivnick, 1986).

Esta narrativa colectiva ofrece una valoración y reconocimiento por las propias historias personales y biográficas de las mujeres, que contradicen las narrativas hegemónicas que asocian la vejez con una etapa improductiva y de declive en la cual las personas solo significan el pasado sin considerar el futuro (Villar y Serrat, 2015). Por lo contrario, las bordadoras dan cuenta de una etapa en la cual se resignifican las experiencias, planifican su futuro y proyectan su continuidad a través de un legado a otras generaciones. Existiría una resistencia al discurso dominante de la vejez como etapa de declive y fragilidad, como también al discurso del envejecimiento exitoso/activo como ideal, pues si bien las mujeres aprecian el hecho de mantenerse saludables y autogestionan su propia salud, reconocen que la vejez no es una etapa idílica o desprovista de problemas, y exploran los cambios y dificultades a los cuales se enfrentan. Así, disputan la vejez exitosa hegemónica y producen una narrativa alternativa que da cuenta de aprendizajes, dificultades, logros y añoranzas.

\section{iv. Vejeces activistas}

[Con las bordadoras] hay mucha, mucha compañía. Es un espacio de sanación; conversamos, reímos, lloramos, fluyen sentimientos de una forma tan extraordinaria (María Cecilia). Es más político, es diferente a otros grupos y a mí me encanta eso; lo que tú no puedes gritarlo lo demuestras en bordados (Gabriela). [Nuestras] historias de vidas muchas son muy tristes y ha sido muy sanador para nosotras este espacio, porque nos hemos encontrado con nuestros pares [...]. Empezamos [el grupo] compañeras que tenían dolores muy profundos y que no lo habían podido hablar nunca, porque no estaban en la instancia de tener otro con quién hablar (Reinalda). Tomamos la decisión de que las que van a integrar el grupo van a ser conocidas a nuestras realidades, porque no se puede asumir que es un grupo que, no sé, yo respeto a los centros de madres, pero no es un grupo que va a bordar; nosotras no bordamos por bordar, sino que bordamos por dejar testimonio de nuestra memoria (Laura). [Finalmente] como que te llena el hecho de poder demostrarle a la gente que nosotras, a pesar de ser mayores, nos damos cuenta de lo que pasa y podemos denunciar la situación, mostrándoselo a otras personas (Aylen). 
En esta narrativa, las mujeres dan cuenta de la importancia que adquiere la Colectiva de las Bordadoras en su experiencia cotidiana. La reconocen como un espacio de soporte emocional y apoyo mutuo, donde el hecho de poder compartir con otras mujeres con trayectorias de vida en común les permite reconocerse y autoidentificarse. Las subjetividades de las mujeres mayores se constituyen como «un proceso social de inteligibilidad mutua» (Cabruja et al., 2000, p. 66) que pueden ser comprendidas desde una perspectiva política. Es decir, las mujeres mayores formaron parte de una generación que vivió un determinado contexto histórico que, para el caso de Chile, estuvo condicionado por la Unidad Popular, la dictadura militar, las consecuencias de la dictadura y los procesos actuales posdictatoriales. Desde esta perspectiva, este tipo de hitos históricos o sociales de gran envergadura pueden propiciar la denominación de un grupo como una generación, pues comparten ciertos marcos históricos y sociales de pensamiento, constituyéndose desde el accionar colectivo y compartido para enfrentar lo social (Reyes, Cornejo, Cruz, Carrillo y Caviedes, 2015).

Algunas investigaciones reportan que, en los procesos asociativos entre mujeres mayores, existiría una suerte de reconocimiento por trayectorias y discriminaciones de género enfrentadas como generación - las que favorecen los vínculos de confianza- y el desarrollo de prácticas de sororidad en la vejez que les permiten afrontar con mayores recursos y empoderamiento esta etapa (Pérez, Rábago y Guzmán, 2018). Estas mujeres asumieron un rol protagónico en la recuperación de la democracia, lo que resulta ampliamente visible por su rol político y público. En este sentido, se ha construido una representación de su resistencia, que les niega la posibilidad de caer y develar sus sentimientos de dolor, atribuyéndoles la capacidad de soportarlo todo, invisibilizando que ellas también fueron «víctimas» de la dictadura (Carrillo, Hernández y Veloso, 2012). En este sentido, la Colectiva se constituye en un espacio terapéutico y sanador, en el que pueden resignificar sus historias y compartir, íntimamente, las experiencias dolorosas, reconociendo su propia trayectoria en el espejo que son sus compañeras. Este acompañamiento surge de la «sororidad» que reivindica que las mujeres —más allá del sentimiento fraterno (frater) — se acompañan y organizan, promoviendo resignificar su coexistencia, desde la reciprocidad y apoyo mutuo como un acto político de hermandad y solidaridad entre mujeres (Pérez et al., 2018).

El ejercicio del activismo en la vejez destaca por sus principios identitarios, donde el cuidado y protección hacia otras generaciones y la mantención de las memorias colectivas serían valores inherentes identificatorios de su acción política (Schwarz, 2019). En este sentido, esta narrativa evidencia la diferenciación 
que las bordadoras realizan con otras agrupaciones que se gestan desde modelos convencionales de envejecimiento, ya en que su acción política se expresa material y simbólicamente en sus bordados. El bordar como acción política da cuenta de una práctica artesanal que se realiza entre mujeres (grupalmente) y que devela que ellas poseen un saber/oficio que heredan de una larga tradición, sin ser un elemento circunstancial de su asociatividad, sino, por lo contrario, identitario. Así, su carácter político y autogestionado les permite un despliegue que contrasta con el ejercicio de participación social institucionalizado y despolitizado que es promovido, en la vejez, por algunos programas sociales (Zubero, 2018). En esta narrativa, se trasciende el foco centrado en las limitaciones, visibilizando la existencia de prácticas y modos de envejecer de las mujeres, quienes amplían los discursos sobre este grupo etario, dando cuenta que su activismo político ofrece otras dimensiones para comprender la vejez desde las mujeres y su pluralidad.

\section{CONCLUSIONES}

En este trabajo, nos propusimos abordar los significados de la vejez de un grupo de mujeres mayores activistas de Chile. A través de sus relatos, construimos narrativas patchwork que ofrecieran miradas difractarias para ampliar la comprensión del envejecimiento femenino. Así, analizamos sus trayectorias y experiencias desde una perspectiva gerontológica feminista, lo que permitió poner atención en las estructuras de opresión que han condicionado la vida de las mujeres, expresándose de un modo particular en la vejez y, a la vez, promovió visibilizar la agencia y las estrategias con las que transitan en esta etapa desde sus experiencias asociativas.

A través de cuatro narrativas ficcionales, ofrecimos una comprensión posicionada y parcial de la vejez de estas mujeres, centrándonos en sus trayectorias asociativas y en sus identidades construidas generacionalmente, lo que nos permitió avanzar en miradas más heterogéneas y contextuales de cara a sus propias experiencias de envejecimiento.

Este estudio fue realizado en un contexto sociosanitario e histórico particular afectado por la pandemia por el coronavirus. Si bien explorar el impacto del COVID-19 no era una de las temáticas iniciales contempladas en este trabajo, esta se constituyó en una dimensión central en los relatos de las participantes y fue considerada por estas como un hito que impactó abruptamente en su experiencia de vejez. La narrativa sexo-genérica da cuenta del carácter diferenciado de la vejez entre hombres y mujeres, y cómo estas se resisten y tensionan el conocimiento 
científico que suele caracterizarlas como frágiles, dependientes y atravesadas por un envejecimiento con mayores problemáticas. Este patchwork de saberes femeninos da cuenta de aprendizajes, adaptaciones, y el desarrollo de procesos subjetivos en esta etapa, que les permiten desplegar sus recursos personales para afrontar los cambios a los que se exponen y que pueden ser transmitidos intergeneracionalmente en la forma de aprendizajes de vida. La narrativa de vejez activista se posiciona como un discurso contrahegemónico que disputa las representaciones sobre las mujeres mayores y también sobre su acción política en la vejez, demostrando el carácter político de sus prácticas, así como las reivindicaciones que continúan desarrollando desde su agrupación. Así, a través de estas narrativas colectivas, se visibilizaron las experiencias de mujeres, sus recursos y resistencias, tensionando los imaginarios de la vejez como una etapa de decadencia, deterioro y fragilidad.

Esta investigación nos invita a pensar que la vejez no es una etapa homogénea y universal, sino más bien que múltiples vejeces pueden emerger y configurarse desde las experiencias compartidas. Consideramos que, al reconocer el valor de la colectividad, la influencia recíproca en la construcción de subjetividades y la capacidad de agencia política de las mujeres mayores, avanzamos en ampliar la comprensión sobre la vejez femenina y ofrecemos otras conceptualizaciones que visibilizan sus contribuciones políticas y sociales. Asimismo, podemos tensionar el conocimiento hegemónico, individualizante y esencialista del ser mujer mayor, contribuyendo ética y ontológicamente a rescatar los discursos y las relaciones fraternas que las propias mujeres tejen a los márgenes. Es decir, reconocemos que las mujeres mayores lideran procesos y cambios en sus respectivos espacios y comunidades.

Estos resultados propusieron desplazamientos al conocimiento hegemónico, que no pretendieron representar el envejecimiento «tal cuál es» sino aportar a su comprensión, desde miradas producidas por subjetividades situadas. Finalmente, aproximarnos a estas narrativas y a la acción política que caracteriza a estas mujeres nos interpela a valorar sus trayectorias de lucha, visibilizar su trabajo y a agradecer los legados y la transmisión de saberes que ofrecen a las siguientes generaciones.

\section{Agradecimientos}

Agradecemos a la Colectiva Bordadoras por la Memoria por su participación compromiso con la investigación.

Este trabajo se ha realizado en el marco del doctorado Persona i Societat en el Món Contemporani de la Universitat Autònoma de Barcelona y el doctorado en Psicología de la Pontificia Universidad Católica de Valparaíso, Chile. 


\section{REFERENCIAS}

Aguierre, R. y Scavino, S. (2018). Vejeces de las mujeres: desafíos para la igualdad de género y la justicia social en Uruguay. Uruguay: Doble Clic.

Arfuch, L. (2007). El espacio biográfico. Dilemas de la subjetividad contemporánea. Buenos Aires: Fondo de Cultura Económica.

Bamberg, M. (2012). Narrative analysis. En H. Cooper, P. M. Camic, D. L. Long, A. T. Panter, D. Rindskopf y K. J. Sher (eds.), APA handbooks in psychology®. APA handbook of research methods in psychology, Vol. 2. Research designs: Quantitative, qualitative, neuropsychological, and biological (pp. 85-102). American Psychological Association. https://doi.org/10.1037/13620-006

Barrett, M. y Lamas, M. (1990). El concepto de diferencia. Debate Feminista, 2. https:// doi.org/https://doi.org/10.22201/cieg.2594066xe.1990.2.1931

Bertaux, D. (2005). Los relatos de vida. Barcelona: Bellaterra.

Biglia, B. (2005). Narrativas de mujeres sobre las relaciones de género en los movimientos sociales (tesis doctoral). Universidad de Barcelona, España.

Biglia, B. y Bonet-Martí, J. (2009). La construcción de narrativas como método de investigación psico-social. Prácticas de escritura compartida. Forum: Qualitative Social Research, 10(1), art 8. Recuperado de https://www.qualitative-research. net/index.php/fqs/article/view/1225/2665

Bravo-Segal S. y Villar F. (2020). La representación de los mayores en los medios durante la pandemia COVID-19: ¿hacia un refuerzo del edadismo? Revista Española de Geriatría y Gerontología, 55(5), 266-271. https://doi.org/10.1016/j. regg.2020.06.002

Burman, E. (2000). Method, measurement, and madness. En Lois Holzman y John Morss (eds.), Postmodern psychologies, societal practice, and political life (pp.49-78). New York: Routledge.

Cabruja, T., Iñíguez, L. y Vázquez, F. (2000). Cómo construimos el mundo: relativismo, espacios de relación y narratividad. Anàlisi: Quaderns de Comunicació I Cultura, 25, 61-94. Recuperado de https://ddd.uab.cat/record/808

Calasanti, T. (2010). Gender and ageing in the context of globalization. In D. Dannefer and C. Phillipson (eds.), The SAGE Handbook of Social Gerontology (pp. 137-149). Londres: SAGE. https://doi.org/10.4135/9781446200933.n10

Carrillo, E., Hernández, E., Bermedo, T. (2012). Los muros del silencio: relatos de mujeres violencias, identidad y memoria. Concepción: Escaparate.

Charpentier, M. y Quéniart, A. (2017). Aging experiences of older immigrant women in Québec (Canada): From deskilling to liberation. Journal of Women and Aging, 29(5), 437-447. https://doi.org/10.1080/08952841.2016.1213111 
Charpentier, M., Quéniart, A. y Jacques, J. (2008). Activism among older women in Quebec, Canada: Changing the world after age 65. Journal of Women \& Aging, 20(3-4), 343-360. https://doi.org/10.1080/08952840801985136

Chazan, M. y Kittmer, S. (2016). Defying, producing, and overlooking stereotypes? The complexities of mobilizing «grandmotherhood» as political strategy. Journal of Women \& Aging, 28(4), 297-308. https://doi.org/10.1080/08952841.2015.1017 428

Comisión Económica para América Latina y el Caribe (Cepal) (2019). La autonomía de las mujeres en escenarios económicos cambiantes. Recuperado de https://www. cepal.org/sites/default/files/publication/files/45032/S1900723_es.pdf

Cornejo, M., Faúndez, X. y Besoain, C. (2017). El análisis de datos en enfoques biográficos-narrativos: desde los métodos hacia una intencionalidad analítica. Forum Qualitative Research Sozialforschung, 18(1), 1-25. Recuperado de http://nbnresolving.de/urn:nbn:de:0114-fqs1701160

Cornejo, M., Mendoza, F. y Rojas, R. (2008). La investigación con relatos de vida: pistas y opciones del diseño metodológico. Psykhe, 17(1), 29-39. http://dx.doi. org/10.4067/S0718-22282008000100004

Del Valle, T. (1999). Procesos de la memoria: cronotopos genéricos. Áreas. Revista Internacional de Ciencias Sociales, 19, 211-225. Recuperado de https://revistas. um.es/areas/article/view/144861

Erikson, E. H., Erikson, J. M. y Kivnick, H. Q. (1986). Vital involvement in old age. Nueva York: Norton.

Espinoza, L. y Rodríguez, A. (2020). Tras el camino andado. Voces, fotografías, relatos e historias de las personas mayores desde el enfoque biográfico-narrativo. En R. Jiménez, C. Mendoza y A. Rodríguez (eds.), Introducción a la metodología cualitativa para el estudio de la vejez y el envejecimiento (pp. 74-91). México: Universidad Autónoma de Tlaxcala.

Esteban, M. L. (2015). La reformulación de la política, el activismo y la etnografía. Esbozo de una antropología somática y vulnerable. Ankulegi: Revista de Antropología Social, 19, 75-93. Recuperado de https://dialnet.unirioja.es/servlet/ articulo? codigo $=5647786$

Freixas, A. (2008). La vida de las mujeres mayores a la luz de la investigación gerontológica feminista. Anuario de Psicologia, 39(1), 41-57. Recuperado de https:// revistes.ub.edu/index.php/Anuario-psicologia/article/view/8393

Freixas, A. (2018). Sin reglas: erótica y libertad femenina en la madurez. Madrid: Capitan Swing.

Freixas, A. (ed.) (2015). Abuelas, madres hijas: la transmisión sociocultural del arte de envejecer. España: Icaria. 
Freixas, A., Luque, B. y Reina, A. (2012). Critical Feminist Gerontology: In the Back Room of Research. Journal of Women and Aging, 24(1), 44-58. https://doi.org/1 $0.1080 / 08952841.2012 .638891$

Gergen, K. y Gergen, M. (1983). Narratives of the self. En Theodore R. Sarbin y Karl E. Scheibe (eds.), Studies in social identity (pp. 225-273). Nueva York: Praeger.

Gonzales, E., Matz-Costa, C. y Morrow-Howell, N. (2015) Increasing Opportunities for the Productive Engagement of Older Adults: A Response to Population Aging. The Gerontologist, 55(2), 252-261. https://doi.org/10.1093/geront/gnu176

Gonzálvez, H. y Lube, M. (2020). Women and aging in social research (1950-2018). Revista Estudos Feministas, 28(1), 1-14. https://doi.org/10.1590/1806-95842020v28n158497

Gonzálvez Torralbo, H. (2018). Género, cuidados y vejez: mujeres «en el medio» del trabajo remunerado y del trabajo de cuidado en Santiago de Chile. Prisma Social, 21, 194-218. Recuperado de https://revistaprismasocial.es/article/view/2445

Gonzálvez Torralbo, H., Larrazabal Bustamante, S. y Lube Guizardi, M. (2020). Envejecimiento, género y cuidados: debates para situar a las políticas públicas. Sociedade e Cultura, 23. https://doi.org/10.5216/sec.v23i.54300

Haraway, D. (1988). Situated Knowledges: The Science Question in Feminism and the Privilege of Partial Perspective. Feminist Studies, 14(3), 575-599. https://doi. org/10.2307/3178066

Haraway, D. (2019). Seguir con el problema. Generar paretesco en el Chthuluceno. Bilbao: Consonni.

Harding, S. (1996). Ciencia y feminismo. Madrid: Morata.

Huenchuán S. (ed.) (2018). Envejecimiento, personas mayores y agenda 2030 para el desarrollo sostenible. Perspectiva regional y de derechos humanos. Santiago de Chile: Comisión Económica para América Latina y el Caribe (Cepal).

Instituto Nacional de Estadísticas (INE). (2018). Síntesis de resultados Censo 2017. Recuperado de https://www.censo2017.cl/descargas/home/sintesis-de-resultados-censo2017.pdf

Katz, S. (2014). What is age studies? Age Culture Humanities: An Interdisciplinary Journal, 1(1), 17- 23. Recuperado de http://ageculturehumanities.org/WP/whatis-age-studies/

Kirkwood, J. (2010). Ser política en Chile, las feministas y los partidos. Santiago de Chile: LOM.

Mazzucchelli, N. (2019). Envejecimiento positivo para Chile: ¿Una vejez sin Estado? Revista Kairós: Gerontologia, 22(3), 25-42. https://doi.org/10.23925/2176901x.2019v22i3p25-42 
Mazzucchelli, N. y Adaos, R. (2019). Género, vejez y violencias: Aproximaciones desde la perspectiva de la interseccionalidad. En C. Rojas, M. Piña y M. G. Olivo (eds.), Envejecimiento \& Cultura: Reflexiones respecto a la vejez y la acción profesional interdisciplinaria junto a personas mayores (pp. 24-77). Talca: UCM.

Ministerio de Salud del Gobierno de Chile (MINSAL) (2020). Casos confirmados en Chile COVID-19. Ministerio de Salud. Recuperado de https://www.minsal.cl/ nuevo-coronavirus-2019-ncov/casos-confirmados-en-chile-covid-19/

Montes de Oca, V. (2011). Viudez, soledad y sexualidad en la vejez: mecanismos de afrontamiento y superación. Revista Kairós Gerontologia, 14 (Especial 10), 73-107. https://doi.org/10.23925/2176-901X.2011v14iEspecial10p73-107

Navarro, M. y Danel, P. M. (2019). Introducción. En P. M. Danel y M. Navarro (eds.), La gerontología será feminista (pp. 29-42). Gualeguaychú: Fundación La Hendija.

Nespereira-García, J. (2014). Los discursos de la pandemia. Nuevas estrategias de comunicación del riesgo en un nuevo contexto sociocultural. Cultura, Lenguaje y Representación. Revista de Estudios Culturales de la Universitat Jaume I, 13(2014), 185-99. http://dx.doi.org/10.6035/clr.2014.13.10.2

Nieve (ed.) (2010). Género, envejecimiento y políticas públicas. Recuperado de http:// dspace.mides.gub.uy:8080/xmlui/bitstream/handle/123456789/1123/Envejecimiento, $\% 20 \mathrm{~g} \% \mathrm{C} 3 \%$ A 9 nero $\% 20 \mathrm{y} \% 20$ pol $\% \mathrm{C} 3 \%$ ADticas $\% 20 \mathrm{p} \% \mathrm{C} 3 \%$ BAblicas. pdf? sequence $=1$

ONU Mujeres (2014). Sesión 2. La organización social del cuidado: identificación de necesidades y escenarios de cuidado. Santiago de Chile: ONU Mujeres Centro de capacitación.

Organización Mundial de la Salud (OMS). (2020). Preguntas y respuestas sobre la enfermedad por coronavirus. Recuperado de https://www.who.int/es/emergencies/diseases/novel-coronavirus-2019/advice-for-public/q-a-coronaviruses

Ortíz, F. y Gonzálvez, H. (2017). Cómo explicar la organización social de los cuidados en Chile: una aproximación al proceso de envejecer. En A. Vera (ed.), Malestar social y desigualdades en Chile (pp. 125-150). Santiago de Chile: Universidad Alberto Hurtado.

Paola, J. (2015). Hacia una perspectiva del trabajo social en el campo gerontológico. En J. Paola, M. Tordó, P. Danel (eds.), Más mayores más derechos. Diálogos interdisciplinarios sobre la vejez (pp. 129-160). Argentina. EDULP.

Pérez, L., Rábago, M., Castillo, G., Schoham, C., Zamora, R. y López, S. (2018). La jubilación y la sororidad femenina: una propuesta de trabajo desde las prácticas colaborativas dialógicas y las narrativas. Nova Perspectiva Sistémica, 27(62), 34-54. https://doi.org/10.38034/nps.v27i62.443 
Pérez, L., Rábago, M., Guzmán, M. y De Jesús, R. (2017). Sororidad en los procesos de envejecimiento femenino. Revistas Diversitas Perspectivas en Psicologías, 14(1), 13-26. https://doi.org/10.15332/s1794-9998.2018.0001.01

Pinazo, S., Torregrosa, M., Jiménez, M. y Blanco, M. (2019). Participación social y satisfacción vital: diferencias entre mujeres y hombres mayores. Revista de Psicología de la Salud, 7(1), 202-234. Recuperado de https://dialnet.unirioja.es/ servlet/articulo?codigo $=6825035$

Piña, C. (1988). La construcción del «sí mismo» en el relato autobiográfico. Chile: FLACSO.

Piña, C. (1999). Tiempo y memoria. Sobre los artificios del relato autobiográfico. Proposiciones, 29, 1-5. Recuperado de http://www.fts.uner.edu.ar/secretarias/ academica/catedras_en_linea/tfoi/mat_catedra/analisis/Pinha.pdf

PNUD (2017). Desiguales: orígenes, cambios y desafíos de la brecha social en Chile. Recuperado de https://www.cl.undp.org/content/chile/es/home/library/poverty/ desiguales--origenes--cambios-y-desafios-de-la-brecha-social-en-/

Ramos, G. (2016). ¡Aquí nadie es viejo! Usos e interpretaciones del Programa Centro del Adulto Mayor-EsSalud en un distrito popular de Lima. Anthropologica, 34(37), 139-169. https://doi.org/10.18800/anthropologica.201602.006

Ramos, M. (2010). Mujeres mayores: nuevos derechos para nuevas realidades. En V. Maquieira (ed.), Mujeres, globalización y derechos humanos (pp. 203-267). Madrid: Cátedra.

Ramos, M. (2017). Envejecer siendo mujer. Dificultades, oportunidades y retos. Barcelona: Bellaterra.

Ramos, M. (2018). Estudio etnográfico sobre el envejecer de las mujeres mayores desde una perspectiva de género y de curso vital. Revista Prisma Social, 21, 75-107. Recuperado de https://revistaprismasocial.es/article/view/2448

Reyes, M. J., Cornejo, M., Cruz, M. A., Carrillo, C. y Caviedes, P. (2015). Dialogía intergeneracional en la construcción de memorias acerca de la dictadura militar chilena. Universitas Psychologica, 14(1), 255-270. https://doi.org/10.11144/ Javeriana.upsy14-1.dicm

Sapriza, G. (2009). Memorias de mujeres en el relato de la dictadura (Uruguay, 19731985). Violencia / cárcel / exilio. DEP. Deportate, esuli, profughe, 11, 64-80. Recuperado de https:/www.unive.it/pag/fileadmin/user_upload/dipartimenti/ DSLCC/documenti/DEP/numeri/n11/07_Dep_11_2009Sapriza.pdf

Sawchuk, D. (2009). The Raging Grannies: Defying Stereotypes and Embracing Aging Through Activism. Journal of Women \& Aging, 21(3), 171-185, https://doi. org/10.1080/08952840903054898 
Schöngut, N. y Pujol, J. (2015). Relatos metodológicos: difractando experiencias narrativas de investigación. Forum Qualitative Research Sozialforschung, 16(2), 44-48. Recuperado de https://ddd.uab.cat/record/132224

Schwarz, C. H. (2019). Collective memory and intergenerational transmission in social movements: The «grandparents' movement» iaioflautas, the indignados protests, and the Spanish transition. Memory Studies. https://doi. org/10.1177/1750698019856058

Serrat, R. y Villar, F. (2020). Lifecourse transitions and participation in political organisations in older Spanish men and women. Ageing and Society, 40(10), 2174-2190. https://doi.org/10.1017/S0144686X19000618

Valdés, T. (1986). El movimiento poblacional: la recomposición de las solidaridades sociales. Santiago de Chile: FLACSO.

Villar, F. y Serrat, R. (2015). El envejecimiento como relato: una invitación a la gerontología narrativa. Revista Kairós: Gerontologia, 18(2), 09-29. https://doi. org/10.23925/2176-901X.2015v18i2p09-29

Wray, S. (2003). Connecting Ethnicity, Agency and Ageing. Sociological Research Online, 8(4), 165-175. https://doi.org/10.5153/sro.866

Wray, S. (2004). What constitutes agency and empowerment for women in later life? The Sociological Review, 52(1), 22-38. https://doi.org/10.1111/j.1467954X.2004.00440.x

Wray, S. (2007). To what extent do ethnic \& cultural diversity influence women's experiences of growing older? En A. Tiihonen y I. Syren (eds.), Age \& Gender, Ikaantyminen ja Sukupuoli edited collection (pp. 1-13). Helsinki: The Age Institute. Recuperado de http://eprints.hud.ac.uk/id/eprint/4734/1/WrayWhat.pdf

Yuni, J. (2019). Prólogo. En P. Danel y M. Navarro (eds.), La gerontología será feminista (pp. 13-23). Gualeguaychú: Fundación La Hendija.

Yuni, J. (septiembre, 2020). Brecha tecnológica generacional y cuidados de la vejez: asimetrías, desigualdades y nuevas dependencias. En G. Casas (presidencia), II Seminario internacional de evidencias de trabajo social en gerontología. El ámbito comunitario. CIETSGE, México.

Zaidi, A., Gasior, K., Hofmarcher, M., Lelkes, O., Marin, B., Rodrigues, R., Schmidt, A., Vanhuysse, P. y Zolyomi, E. (2013). Active Ageing Index 2012 Concept, Methodology and Final Results. Recuperado de https://www. researchgate.net/publication/256008735_Project_Active_Ageing_Index_ AAI'_Active_Ageing_Index_2012_Concept_Methodology_and_Final_Results

Zubero, I. (2018). Envejecimiento activo y participación política. Aula abierta, 47(1), 21-28. https://doi.org/10.17811/rifie.47.1.2018.21-28 